\title{
Electrocardiographic Changes in Hypothermia
}

Eleftheria Kampouri, M.D. Julien Vaucher, M.D.

Lausanne University Hospital Lausanne, Switzerland

eleftheria-evdokia.kampouri@chuv.ch

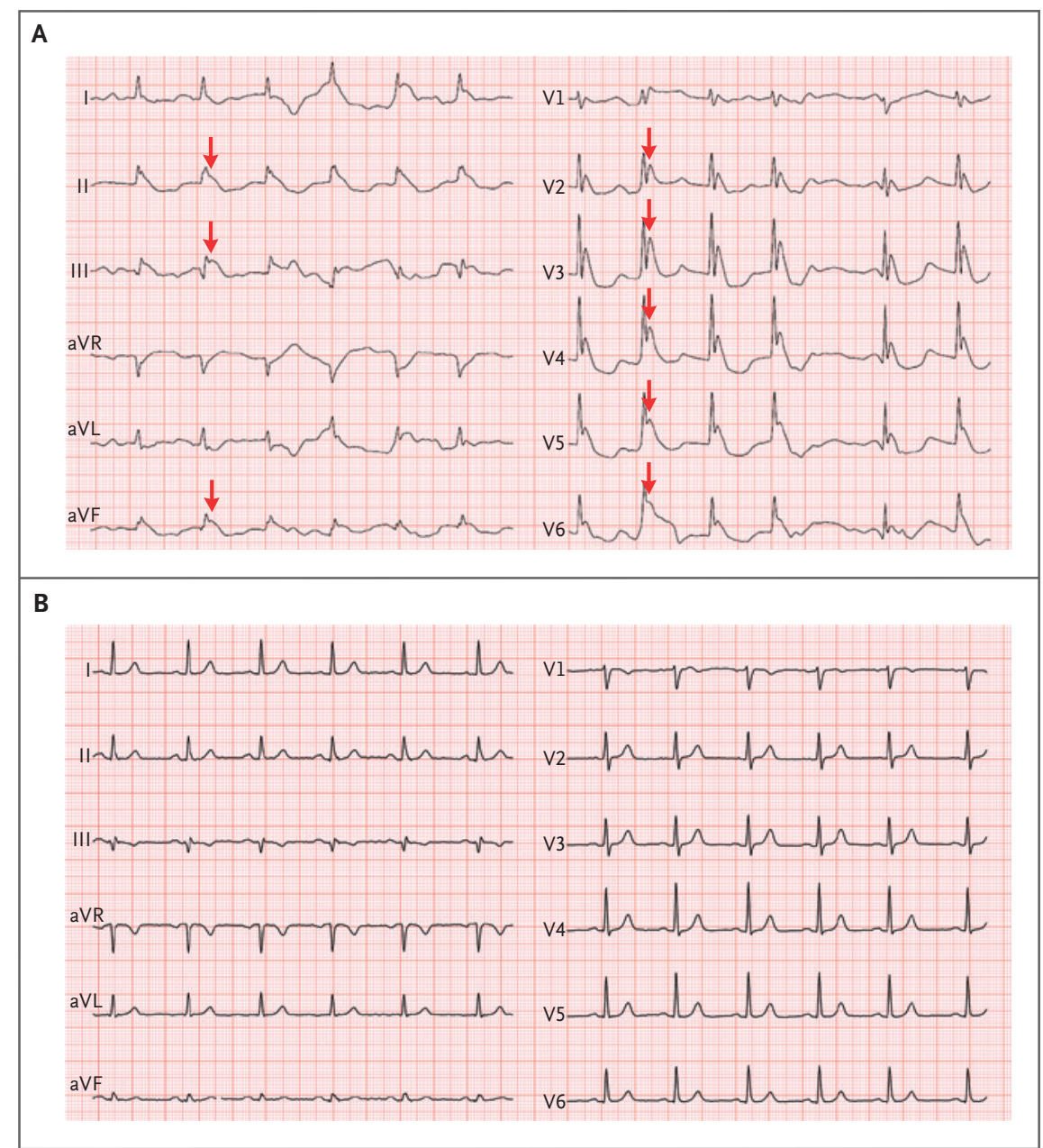

76-YEAR-OLD WOMAN WITH A HISTORY OF DEMENTIA AND CORONARY HEART DISEASE WAS BROUGHT TO the emergency department after she had been found lying outdoors for an undetermined period; her core body temperature was $26^{\circ} \mathrm{C}\left(78.8^{\circ} \mathrm{F}\right)$ at presentation. An electrocardiogram showed prominent Osborn waves, also known as J waves (Panel A, arrows), along with prolonged QRS duration and corrected QT interval. Laboratory testing revealed a serum potassium level of $5.7 \mathrm{mmol}$ per liter (reference range, 3.5 to 4.6), a creatine kinase level of $1230 \mathrm{U}$ per liter (reference range, 25 to 190), and a serum $\mathrm{pH}$ of 7.19. The serum calcium level was normal. Hypothermia induces an increase in the activity of the cardiac transient outward potassium current, which is more prominent in the epicardium than in the endocardium. This heterogeneous distribution of potassium current results in J waves that are typically observed in the inferior and lateral precordial leads, as seen in this patient. The magnitude of the J waves may generally be associated with the degree of hypothermia. In addition to hypothermia, J waves have been associated with conditions that can occur in normothermia (e.g., hypercalcemia, the Brugada syndrome, and neurologic injury). After the patient was rewarmed for 12 hours, the J waves disappeared, and the QT interval and QRS duration normalized (Panel B).

DOI: 10.1056/NEJMicm1704534

Copyright (C) 2018 Massachusetts Medical Society.

This article was updated on February 1, 2018, at NEJM.org. 